\title{
Update on surgical management of complex macular holes: a review
}

\author{
Mohd-Asyraaf Abdul-Kadir *iD and LikThai Lim
}

\begin{abstract}
Modern surgical interventions effectively treat macular holes ( $\mathrm{MHs}$ ) more than $90 \%$. Current surgical treatment for MHs is pars plana vitrectomy with epiretinal membrane, internal limiting membrane (ILM) peeling, gas endotamponade, and prone posturing postoperatively. However, a small subset of MHs imposes challenges to surgeons and frustrations on patients. A narrative review was performed on the surgical treatment of challenging $\mathrm{MHs}$ including large and extra-large MHs, myopic MHs with or without retinal detachment, and chronic and refractory MHs. There are robust data supporting inverted ILM flap as the first-line treatment for large idiopathic MHs and certain secondary MHs including myopic MHs. In addition, several studies had shown that ILM flap manipulations in combination with surgical adjuncts increase surgical success, especially in difficult MHs. Even in eyes with limited ILM, surgical options included autologous retinal graft, human amniotic membrane, and creation of a distal ILM flap that can assist in MH closure even though the functional outcome may be affected by the MH chronicity. Despite relative success anatomically and visually after each technique, most techniques require a long-term study to analyze their safety profile and to establish any morphological changes of the MH plug in the closed MHs.
\end{abstract}

Keywords: Macular hole, Vitrectomy, Vitreoretinal disease, Internal limiting membrane peeling

\section{Introduction}

Macular holes (MHs) were once considered non-treatable and an $\mathrm{MH}$ was first described in 1869 from a traumatic origin [1]. MH is characterized by a vertical defect in the neurosensory retinal anatomy particularly in the foveal region that extends from the internal limiting membrane (ILM) to the retinal pigment epithelium (RPE) and it affects the central vision and causes metamorphopsia [2]. MHs are predominantly idiopathic (primary) with higher prevalence with increasing age and in females. Its estimated annual incidence is up to 8.69 eyes in 100, 000 population [3, 4]. Secondary MHs are attributed to but not limited to high myopia, trauma, proliferative diabetic retinopathy, and various retinal pathologies.

*Correspondence: akmasyraaf@unimas.my

Present Address: Department of Ophthalmology, Universiti Malaysia

Sarawak (UNIMAS), Kota Samarahan, Malaysia
Current surgical techniques successfully close majority MHs greater than $90 \%$ with remarkable visual acuity gain; however, small percentages of $\mathrm{MHs}$ have a higher risk of initial surgical failure [5, 6]. Large MHs, MHs with a basal diameter of $>400 \mu \mathrm{m}$, are likely to have a flat-open closure or flat MH margins with bare RPE configuration with unsatisfactory visual prognosis despite closure [710]. The 5-Year Manchester Large Macular Hole Study found a higher success rate between 91 and $98 \%$ of surgical closure for large $\mathrm{MHs}$ with diameter in the range of $400-649 \mu \mathrm{m}$ while MHs with diameter $650-1416 \mu \mathrm{m}$ only achieved $76 \%$ [11]. Another study reported that the rate of $\mathrm{MH}$ closure was only $56 \%$ in eyes with a large $\mathrm{MH}$ of $>400 \mu \mathrm{m}$ and about $10 \%$ of the closed MHs reopened after 6 months [12]. Moreover, surgical success in $\mathrm{MH}$ repair was found to be more than $90 \%$ within one year after the onset of the symptoms and lowered to $47.4 \%$ after 1 year [13]. Minimal visual gain can still be achieved after closing chronic MHs although this is associated with the MH duration [14]. original author(s) and the source, provide a link to the Creative Commons licence, and indicate if changes were made. The images or other third party material in this article are included in the article's Creative Commons licence, unless indicated otherwise in a credit line to the material. If material is not included in the article's Creative Commons licence and your intended use is not permitted by statutory regulation or exceeds the permitted use, you will need to obtain permission directly from the copyright holder. To view a copy of this licence, visit http://creativecommons.org/licenses/by/4.0/. The Creative Commons Public Domain Dedication waiver (http://creativeco mmons.org/publicdomain/zero/1.0/) applies to the data made available in this article, unless otherwise stated in a credit line to the data. 
Meanwhile, highly myopic MHs have been identified as another risk factor for failed $\mathrm{MH}$ repair, with at least $26 \mathrm{~mm}$ of axial length (AL) or refraction more than -6 Diopter in spherical equivalent [15]. Anatomical success in myopic $\mathrm{MH}$ repair declined with the increasing AL, from 91.7\% (AL: $26-29.9 \mathrm{~mm}$ ) to $0 \%$ in eyes with $\mathrm{AL}>30 \mathrm{~mm}$ [16]. The rigid ILM relatively resisted the anterior-posterior traction from the presence of posterior staphyloma and increasing $\mathrm{AL}$ and contributed to the formation of myopic MH [17]. Retinoschisis can also be seen commonly in persistent MHs and may contribute to late retinal detachment (RD) in unsuccessful surgical repair [18]. Other mechanisms that have been reported to cause surgical failure include residual tractional force from epiretinal membranes (ERMs), non-compliance with prone posturing, suboptimal intraocular tamponade, and postoperative cystoid macular edema [19-23].

There has been an increasing advent of more advanced and complex surgical techniques to treat these subsets of MHs with less favorable outcomes including persistent and recurrent MHs, high myopia with $\mathrm{MH}$ with and without RD, large MHs $>650 \mu \mathrm{m}$, and chronic MHs [24]. Therefore, this article aims to comprehensively review the available techniques to tackle these challenging MHs.

\section{Types of MH closure}

It is also essential to identify the closure patterns of fullthickness macular holes (FTMHs) as there has been a correlation between the postoperative $\mathrm{MH}$ morphological features and the visual prognosis. An $\mathrm{MH}$ configuration with Type 2 or W-type has a less favorable visual outcome even though the MH is practically closed [10, 25]. Hillenkamp et al. also described refractory FTMH with subretinal fluid cuff as more likely to achieve anatomical success and improvement of best-corrected visual acuity (BCVA) following reoperation [26] (Table 1).

\section{Mechanism of $\mathrm{MH}$ repair}

The early work in closing MHs was documented in the early 1990s, which included pars plana vitrectomy (PPV), removing adherent cortical vitreous, peeling of ERM, and a total gas-fluid exchange with long-acting intraocular gas tamponade. The goals were to relieve the anteriorposterior and tangential tractions on the vitreomacular interface and to appose the MH edges closer with gas endotamponade [22]. Surgical reattachment of detached macular could potentially improve the patient's visual acuity as seen in $73 \%$ of the functional success rate postoperatively in $30 \mathrm{MHs}$ that were closed [22].

With the advent of modern imaging modalities, the pioneering initiative by Kelly et al. has been modified into more sophisticated techniques to treat refractory $\mathrm{MHs}$ including ILM peeling, inverted ILM (i-ILM), i-ILM flap, pedicle ILM flap, retracting ILM door, autologous free ILM flap, non-ILM grafts, human amniotic membrane, hAM graft, adjuvant chorioretinal adhesives, and experimental mesenchymal stem cells (MSCs). Other methods that have been investigated were relaxing arcuate retinotomy, subretinal infusion, and hydrodissection.

Gliosis can be induced by peeling the ILM alone by activation of Müller cell fragments in the ILM. Thus by inverting the ILM flap over the MH, it acts as a scaffold for Müller cell tissue proliferation and may induce gliosis inside the retina and on the ILM surface, followed by $\mathrm{MH}$ filling [8]. Besides, an animal study had shown that Müller cells could produce photoreceptors following a toxic injury [27]. In addition, the ILM flap provides a barrier to prevent the vitreous fluid from entering the $\mathrm{MH}$, thus allowing subretinal fluid reabsorption via the RPE [28]. In ILM peeling, the intraocular air or gas tamponade also provides the scaffold or creates a partition between the RPE and the fluid while enforcing further stabilization in the i-ILM flap [29].

The common practice for FTMH management is PPV with posterior hyaloid removal, ERM (if present) and ILM peeling, gas tamponade, and prone posturing postoperatively [30, 31]. Cheng et al. elucidated that peeling of ERM increases the rate of anatomical closure to $67 \%$ compared to $35 \%$ in the non-ERM peel group and the presence of excessive ERM postoperatively contributed to the reopening of MHs [32]. A meta-analysis in 2016 reported that ILM peeling was associated with a statistically significant reduction in the likelihood of $\mathrm{MH}$ reopening from $7.12 \%$ to $1.18 \%$ and significantly lower reoperation rates according to a large cross-sectional study $[33,34]$. Another study demonstrated no significant difference between short-acting sulfur hexafluoride, $\mathrm{SF}_{6}$, and long-acting $\mathrm{C}_{3} \mathrm{~F}_{8}$ in the hole closure rate, regardless of the hole size, stage, chronicity, or intraoperative dye used [35-37]. $\mathrm{C}_{3} \mathrm{~F}_{8}$ may add benefit to the reoperation of persistent and recurrent MHs [36]. Facedown posturing offers potential superior visual acuity gain, although with no additional advantage in anatomical closure in large MHs [38].

\section{ILM peeling}

The ILM peeling technique was first reported in a series of 39 eyes with FTMH with closure rate at $92 \%$ of eyes and $77 \%$ of closed $\mathrm{MH}$ displayed visual improvement at least two lines [39]. According to a Cochrane review and randomized controlled trials (RCTs), ILM peeling in idiopathic FTMHs confers superior benefits in increasing the likelihood of primary anatomical closure with additional benefits from dye-assisted peeling namely indocyanine green (ICG) and trypan blue (TB) [40, 41]. It lowers the risk of reoperation [5, 42], and extensive meta-analysis 
studies reported a better success rate in anatomical closure in the ILM peel group of 94.1-96\% particularly in Stage III and IV and chronic MHs [43, 44]. However, there was no difference in the primary visual acuity improvement in peel and non-peel groups at six months [5], consistent with the findings of other trials [42, 45].

A long-term study has ascertained the safety of ILM peeling as the absence of late reopening of successfully closed MHs with significant improvement of the median BCVA from the baseline of $20 / 100$ to $20 / 32$ postoperatively. The authors also claimed that the visual acuity changes were independent of the duration of symptoms, the MH stages, or the number of operations [46].

$\mathrm{Al}$ Sabti and colleagues had successfully demonstrated closure of two extra-large MHs, measured at 1147 and $773 \mu \mathrm{m}$ with extended ILM peeling up to the arcades and both eyes gained improvement in their postoperative visual function [47]. In MHs that failed to resolve after ILM peel with dye-assistance, enlarging the ILM-rhexis from prior peel may provide additional benefits. However, an early study on reoperation of persistent $\mathrm{MHs}$ that failed initial PPV showed a lower closure rate and poor visual prognosis despite closed MHs after secondary surgery [48]. Reoperation achieved varying closure rates from 46.7 to $68.9 \%$ in refractory MHs, which included enlarging ILM peel up to the vascular arcade and the posterior fundus to release further tangential traction on the $\mathrm{MH}$ $[49,50]$. Meanwhile, metamorphopsia was alleviated with statistically significant improvement in visual acuity in closed MH cases, which was likely correlated to the alleviation of asymmetric elongation of foveal tissue [51].

ILM peel is associated with mechanical trauma to the retinal nerve fiber layer, including dissociated optic nerve fiber layer (DONFL) [52-54]. These changes appear on optical coherence tomography (OCT) as dark striae in areas of the previously peeled site in autofluorescence imaging and these were likely due to the damage to Müller cells from ILM peel that causes dehiscence of the optic nerve fiber bundles [55]. The formation of DONFL could also be a healing response following retinal nerve fiber layer (RNFL) dehiscence after ILM peel [19]. Although they potentially disappear within one to three months postoperatively, few authors had documented these changes in their cohorts from six up to 12 months with no definitive evidence of loss of visual function or microperimetry changes $[19,53,55]$.

\section{i-ILM Flap}

Michalewska et al. performed PPV with i-ILM flap overlying MHs in large idiopathic MHs as per Fig. 1a and compared it to the conventional PPV with ILM peeling. The i-ILM flap group had $98 \%$ closure rates with a better functional outcome while the standard PPV group only achieved $88 \%$ anatomical success after the first surgery. A flat-open configuration was only seen about $2 \%$ in the former group compared to $19 \%$ in the latter [8]. Maneuvering the ILM flap intraoperatively imposes challenges to the surgeons as they face a steep learning curve to ensure effective surgical outcomes.

However, the original i-ILM flap method resulted in a multilayered membrane as identified in the postoperative imaging. Therefore, a single-layer inverted flap was introduced to create a more physiological and regular structure for gliosis and $\mathrm{MH}$ closure [56]. Figure $1 \mathrm{~b}$ depicts variations of single-layer flaps by Shin et al. and Michalewska et al. $[19,57]$. The single-layer i-ILM flap technique resulted in $83 \%$ closure with improved mean visual acuity [57]. Temporal i-ILM flap by Michalewska et al. achieved similar anatomical closure and visual improvement compared to the conventional i-ILM method for Stage IV MHs [19]. Another study reported that a higher success rate with the vertical flap was likely secondary to gravitational and more powerful inward tangential forces to close the MHs in a study that compared various sizes and locations of the i-ILM flap [21].

Few authors had incorporated adjuncts during surgery to stabilize and tamponade the flap in situ. Shin et al. used perfluoro-n-octane (PFC) before the fluid-gas exchange to prevent the displacement of the flap and for its repositioning if required [57], and others had included ocular viscoelastic device (OVD) and autologous blood clot $[20,58]$.

Minimizing the peeling area is also beneficial for reducing iatrogenic trauma in the papillomacular bundle area. DONFL was only observed localized to the peeled ILM region and these studies had proven that the large flap was not necessary for the i-ILM technique $[19,59]$. Temporal i-ILM flap also caused less microvascular changes particularly in the deep capillary plexus and retinal sensitivity compared to ILM peeling [60, 61]. However, another study compared temporally versus nasally harvested i-ILM flaps in MHs $<600 \mu \mathrm{m}$ and found significantly higher "deep inner retinal dimples" in the former group, $35 \%$ than the latter, and $5 \%$ with no correlation to the BCVA outcomes. Significant temporal macular thinning was greater in the temporal group and negatively correlated with BCVA. All eyes otherwise achieved 100\% closure with $92 \%$ demonstrated U-type closure and ellipsoid zone (EZ) restoration was observed in 62\% [58].

Folding i-ILM flap into $\mathrm{MH}$ also creates a multilayered appearance and it might hinder realignment of the outer retinal layers and thus vision improvement [62]. Few studies had recommended covering the MH with i-ILM flaps instead of "inserting" the flap into the MH due to potential trauma to the RPE in the fovea. An i-ILM flap covering $\mathrm{MH}$ showed significantly better BCVA, retinal 

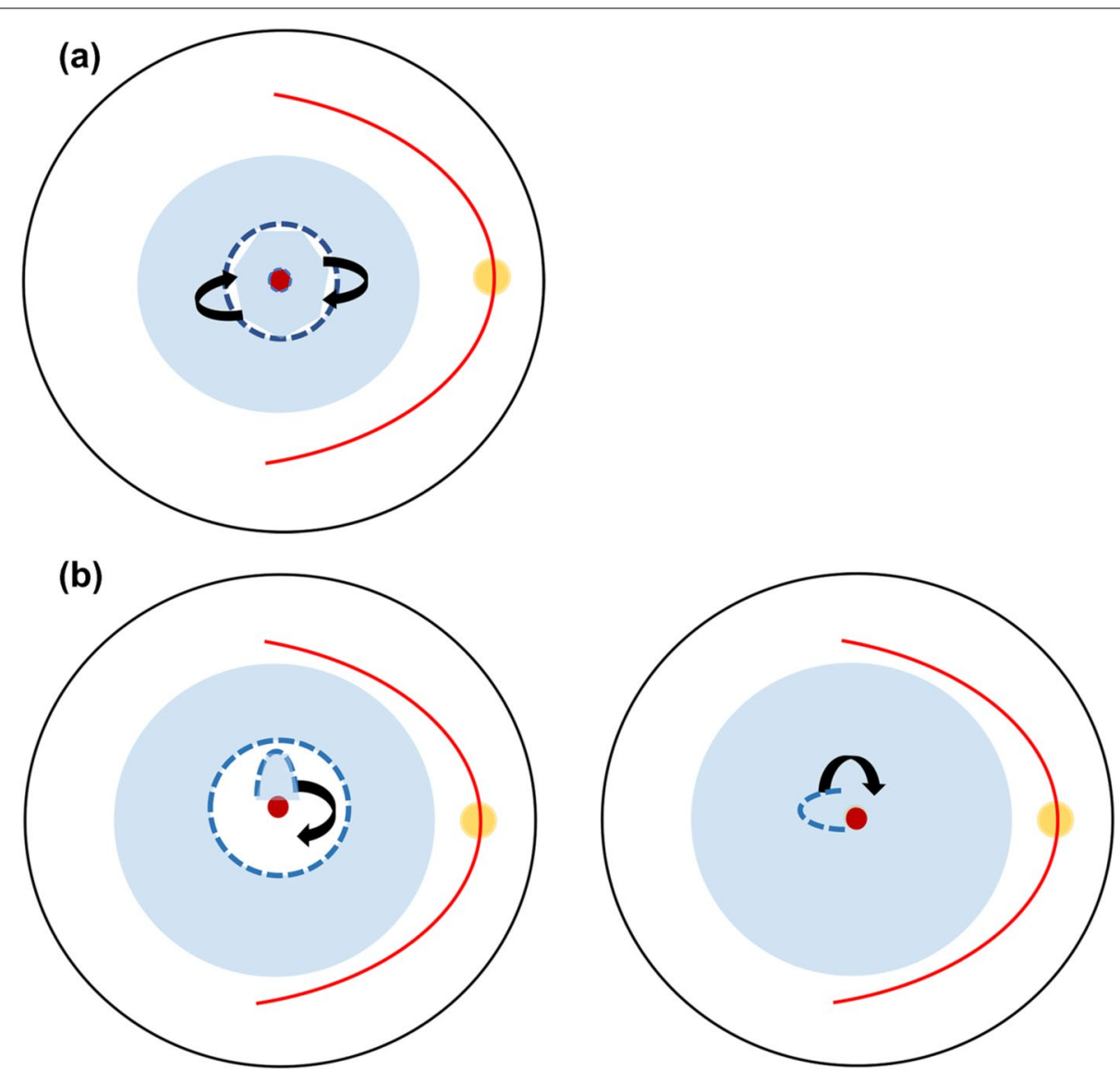

Fig. 1 a i-ILM Flap by Michalewska et al. Following core vitrectomy, ILM was stained with TB and ERM was removed if present. Approximately 2 DD of ILM was peeled circumferentially and left attached to the edges of the MH. The peripheral ILM was trimmed and the central remnant of ILM was massaged until inverted over the MH. Subsequently, air-fluid exchange was performed with intraocular gas tamponade. Patients were advised to stay in a position that allowed them to see the air bubble in their central vision for 3-4 days. b Left—Shin et al. stained the ILM using brilliant blue-G (BBG) and removed the surrounding ILM but a 1 DD-sized flap superiorly to MH. PFC was injected over the flap for stabilization and repositioning if needed. Right-Michalewska et al. performed temporal i-ILM flap and about 2 DD of ILM was peeled temporally to the MH edge. The flap was then inverted over the MH until adequate coverage was obtained

sensitivity, and fixation stability compared to the "insert" group in idiopathic MHs repair [63]. The cover technique also yielded better postoperative visual gain as complete recoveries of the EZ and external limiting membrane (ELM) defects were seen even though both insert and cover groups achieved similar anatomical success [64]. Furthermore, Park et al. recommended i-ILM for superior visual acuity gain as no complete resolution of EZ and ELM defects was observed in the ILM insertion group in closing large MHs $>500 \mu \mathrm{m}$ while i-ILM flap yielded better recovery of layers of photoreceptors [65].

Rizzo et al. analyzed 620 eyes with idiopathic or myopic MHs and found that vitrectomy with an i-ILM flap yielded statistically significant anatomical closure and

Table 1 Different types of configurations of MH closure

\begin{tabular}{lll}
\hline Authors & Imai et al. [10] & Kang et al. [25] \\
\hline Closure patterns of FTMH & $\begin{array}{l}\text { U-type: normal foveal contour } \\
\text { V-type: Steep foveal contour } \\
\text { W-type: Foveal defect of the neuro- } \\
\text { sensory retina }\end{array}$ & $\begin{array}{l}\text { Type 1: closed MH without foveal defect of the neurosensory retina } \\
\text { tively although the rim of the MH is attached to the RPE and the cuff is } \\
\text { flattened }\end{array}$ \\
\hline
\end{tabular}


improvement in BCVA compared to standard PPV with the ILM peel group. The anatomical closure rate in large FTMH was $96 \%$ versus $79 \%$ and in highly myopic MHs, it was $88 \%$ versus $39 \%$ between the i-ILM and ILM peel group. Overall, the i-ILM group had significantly better mean postoperative BCVA, $0.43 \log$ MAR than $0.52 \log$ MAR in the ILM peel group [66]. A systematic review demonstrated that the i-ILM flap method even achieved significantly better anatomical and functional success than ICG-assisted ILM peeling [67]. Meanwhile, the evidence for larger MHs was contradicting as Manasa et al. observed that i-ILM is more superior anatomically and functionally to ILM peel in MHs $>600 \mu \mathrm{m}$ [68] but additional trials did not demonstrate any difference between both surgical groups in these larger MHs [69, 70]. Another study did not find any difference in anatomical closure between the standard ILM peel and i-ILM flap techniques in medium-large $(400-500 \mu \mathrm{m})$ and extralarge MHs $(>550 \mu \mathrm{m})$ but the authors recommended that the $\mathrm{i}$-ILM flap is more effective in extra-large MHs as the group yielded $100 \%$ anatomical closure postoperatively compared to ILM peeling [9].

In myopic MHs with RD, an i-ILM flap is associated with significantly better $\mathrm{MH}$ closure and retinal reattachment compared to the ILM peel group although there was no difference in the postoperative BCVA in both groups. It reduced the risk of recurrent RD as unclosed MHs may increase the risk of retinal re-detachment [71]. All myopic MHs with RD had successful retinal reattachment in both ILM peel and i-ILM flap groups but significantly better recovery rates of the ELM and EZ layers were observed in the latter group [72]. The i-ILM flap technique also closed all myopic $\mathrm{MH}$ without RD and enhanced foveal architectural regeneration as seen within 12 months postoperatively with an associated mean visual acuity gain of $0.64 \log$ MAR [73]. A meta-analysis demonstrated that i-ILM flap has a significantly higher and more superior anatomical closure rate in each subgroup of MHs, large idiopathic MHs and myopic MHs with or without RD [74].

Long-term analysis showed mixed evidence of the efficacy and safety of i-ILM. The visual recovery in the i-ILM flap group was significantly higher at 3 months compared to ILM peeling but no difference was identified at a longer follow-up more than 6 months while performing better anatomical success than the ILM peel group [75]. Another study had shown a promising impact of i-ILM flap in treating large MHs as the recovery of the ELM had been identified as early as 3 months and yielded significantly higher foveal restoration than the ILM peel group [76]. Nevertheless, there is a strong correlation between the BCVA and the integrity of the EZ layer, independent of the presence of the ILM flap [77]. However, the
i-ILM flap group showed lower recovery rates of the EZ and ELM and required a longer recovery period of the ELM postoperatively than the ILM peel group. Thus, the changes in BCVA gain are significantly smaller in the i-ILM group, and the authors suggested that there is a limited role of i-ILM flap in managing refractory $\mathrm{MHs}$ [78]. A recent publication studied the changes of temporal i-ILM flap at 1 month and 6 months postoperatively and found that ILM flap contracted significantly especially in the younger cohort and one eye had more than $20 \%$ shrinkage and required reoperation [79].

\section{SWIFT}

In Fig. 2a, Tabandeh et al. modified i-ILM flap to SWIFT or "superior wide-base internal limiting membrane flap transposition" for complicated MHs including refractory, chronic, and highly myopic MHs with previous ILM removal. In the study, 17 complicated MHs were included in the retrospective series with a mean basal diameter (MBD) of $899.4 \mu \mathrm{m}$ and the SWIFT method yielded $94 \%$ closed MHs and improved mean VA from baseline 0.88 to $0.54 \log$ MAR at the final visit. By using the ICG fluorescence imaging, the ICG-stained SWIFT flap was visualized postoperatively and $82 \% \mathrm{MHs}$ had full coverage of the flap, whereas the remaining $\mathrm{MHs}$ had partial or no coverage. However, one $\mathrm{MH}$ was closed in eyes that were not completely covered by the flap [80].

Tabandeh highlighted that the cross-sectional view of OCT may not be able to distinguish the flap's location and its status. The author described that the "en-face" visualization of ICG-stained SWIFT flap using confocal laser imaging at $795 \mathrm{~nm}$ had shown variable degrees of ICG hyper-fluorescence, which indicated to the position of the flap and the MH coverage, whereas the hypo-fluorescence area reflected the flap harvest site. As the ICG signal may fade, the author recommended ICG imaging between two and four months, postoperatively, after the resolution of gas endotamponade [81].

\section{Pedicle ILM transposition}

$\mathrm{Hu}$ et al. argued that a single-layer and non-inverted flap is more physiological than the i-ILM flap technique as theoretically the glial proliferation and the macular closure are more favorable if the retinal side of the flap covers the $\mathrm{MH}$ as per Fig. 2b. The method achieved $91.7 \%$ of V-type closure in large $\mathrm{MHs}$ and the closure was observed as early as day 1 postoperatively in six SO-filled eyes. The mean BCVA was significantly improved from baseline at $1.23 \log$ MAR to $0.67 \log$ MAR postoperatively and retinal sensitivity and multifocal electroretinogram (mERG) responses were 

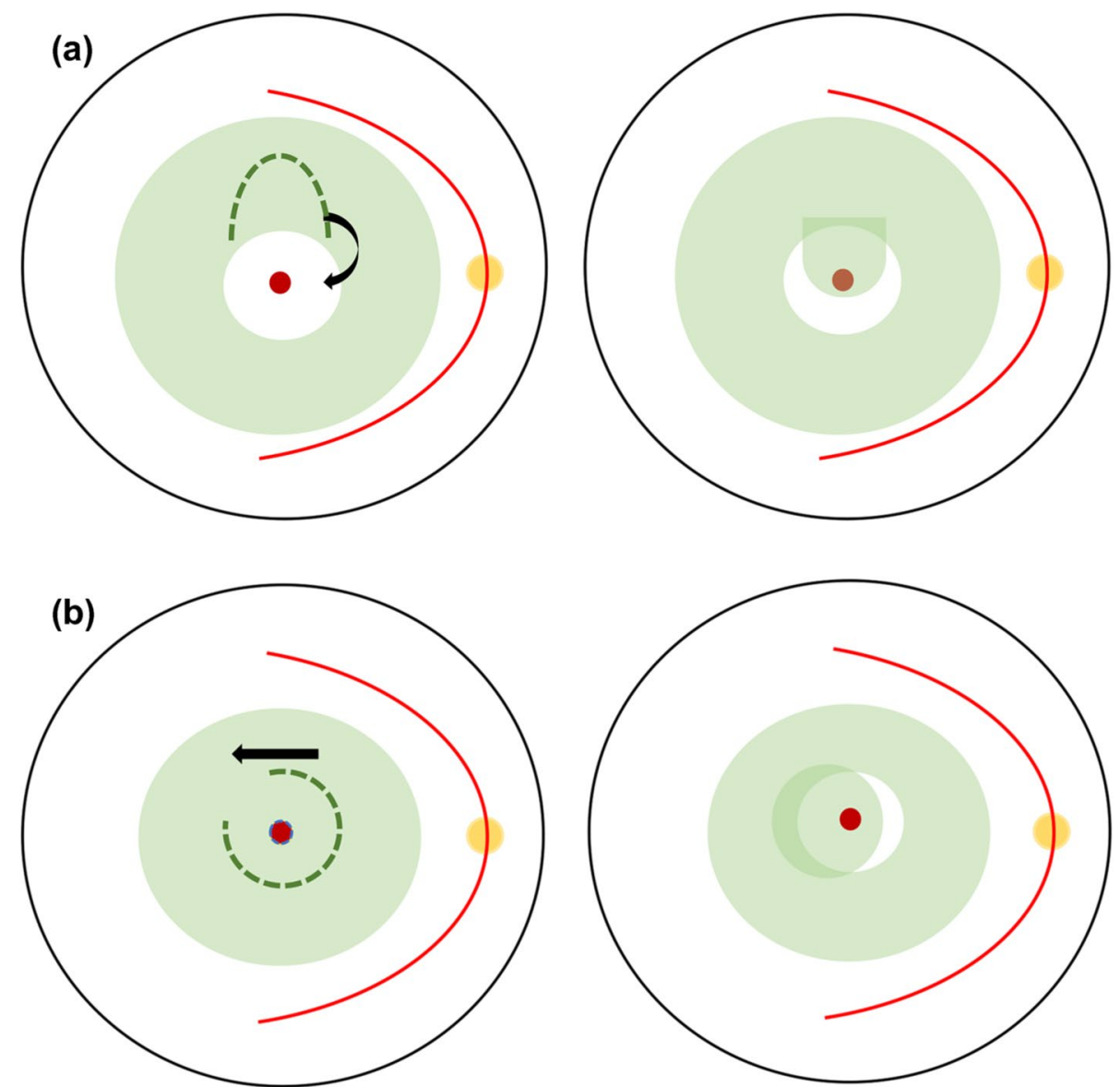

Fig. 2 a Tabandeh et al. performed a distal superior ILM flap from the MH and transposed it over the MH. b Formation of pedicle ILM by Hu et al. PFCL was injected to protect the MH and the exposed RPE before staining the ILM with ICG or BBG. Then the ILM peeling was performed circumferentially around the MH for at least 2 DD and left attached to the superior temporal retina. The pedicle ILM then rotated and transposed over the $\mathrm{MH}$, with its nasal part fully covered the $\mathrm{MH}$ while stabilised and flattened under a larger bubble of PFCL followed by air-fluid-PFCL exchange. The authors chose either SO or autologous whole blood with C3F8 gas to prevent displacement of the ILM pedicle transposition and patients were advised to prone-posturing for 3 days

significantly improved in correlation to attached ILM plug on the macular and restoration of the retinal layers [82].

A preliminary study demonstrated the pedicle ILM transposition as a primary method in successfully closing three very large MHs with a minimum diameter $>700 \mu \mathrm{m}$ out of four eyes. One eye achieved U-type with one line BCVA improvement and two eyes yielded V-type closure with 1-2 lines of BCVA gain [83]. While the authors argued that the technique is not feasible in eyes that had undergone prior ILM peel, the pedicle ILM transposition method had been used in two refractory MHs when there was no ILM available around the MHs. The pedicle was created by peeling the remaining inferior ILM toward superiorly, which then transposed over the $\mathrm{MH}$ while still attached to the superior margin of $\mathrm{MH}$. The method eliminates the risk of flap loss observed in autologous free ILM transplantation for managing recurrent and persistent MHs [84]. However, some authors reported that the flap contracted over time and reopened the $\mathrm{MH}[85]$.

\section{Retracting ILM door}

A novel technique was introduced that successfully treated two myopic MH with and without RD by creating a hinged retracting ILM flap as per Fig. 3. This is to relax the rigid ILM seen in highly myopic eyes and the retracting flap over the $\mathrm{MH}$ will provide the scaffold needed for the proliferation of cells and the migration of photoreceptors. Both eyes gained remarkable visual gain from counting fingers and $20 / 80$ to $20 / 50$ postoperatively. This technique also eliminated excessive manipulation and prevented the displacement of ILM 


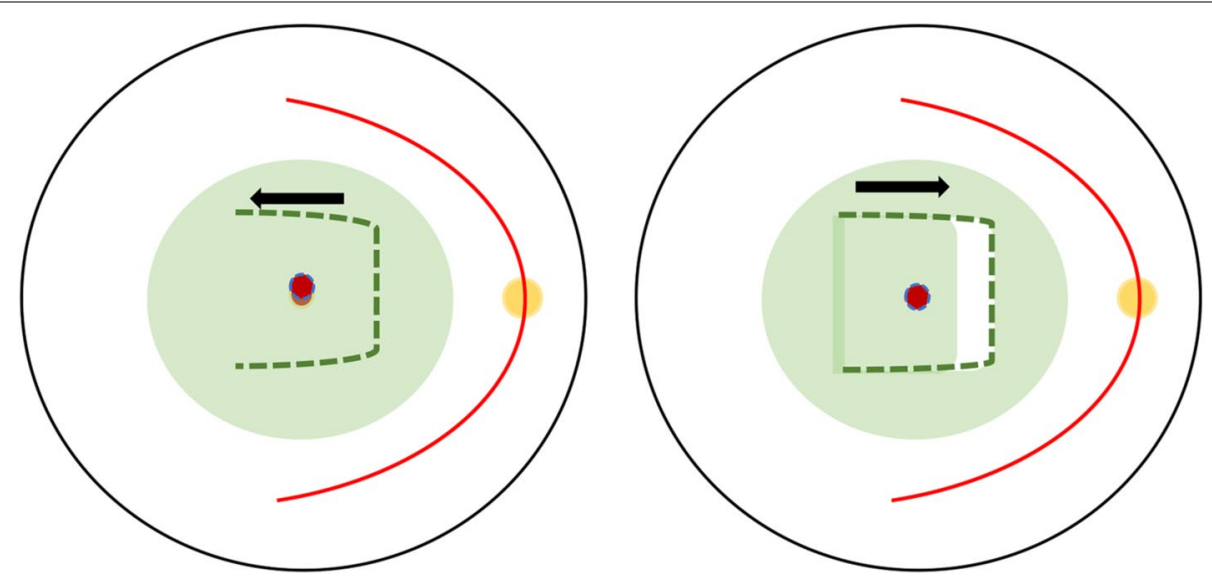

Fig. 3 ILM retracting door. The ILM was stained using indocyanine green (ICG) and a large flap was created starting nasally to temporally, including over the fovea and the $\mathrm{MH}$ area. The flap which now hinged temporally then freely draped over the $\mathrm{MH}$ and thus the nasal ILM flap covered the MH

flap as well as preserving ILM unlike the i-ILM flap, which could be potentially beneficial in cases with thin retinae and for future use if required [17]. This method also maintains the original orientation of ILM and Müller cells although its long-term profile remains unkn own.

\section{Autologous ILM free flap}

Surgical failure in MHs that already had ILM peel somehow may limit further potential treatments in the refractory MHs. Morizane et al. introduced autologous transplantation of the ILM in cases with refractory $\mathrm{MH}$ that failed to close after previous ILM removal. The authors harvested a similar diameter of ILM flap to the $\mathrm{MH}$ and during the intraoperative period, the infusion was turned off to prevent the turbulence from displacing the free flap, and then an OVD was injected overlying the flap and $\mathrm{MH}$. Even in complicated cases including traumatic and myopic MHs, the technique achieved $\mathrm{MH}$ closure in $90 \%$, and $80 \%$ had improvement in visual acuity by more than the logarithm of the minimum angle of resolution, logMAR 0.20 [86].

Similarly, autologous ILM free flap yielded 93.3\% closure in recurrent primary MHs with a significantly better functional outcome at 3 months than the control ILM enlargement peel group. The former group also achieved lesser defects of the inner and outer retinal segments [87]. Multiple free ILM flaps and double ILM layered insertion into highly myopic $\mathrm{MH}$ with $\mathrm{RD}$ also achieved superior closure rate and assisted retinal reattachment in all cases versus ILM peeling alone [88, 89]. An imaging study found that the autologous ILM free flap promoted prolonged glial proliferation that led to the closure of large or refractory MHs with evidence of foveal depigmentation and fibroplasia and partial regeneration of EZ and ELM defects. These foveal changes however did not affect the visual outcome but further study is needed to elucidate the safety profile of this technique [90].

Managing the autologous ILM free flap intraoperatively can be challenging as poor visual of the flap despite adequate dye staining. Dai et al. injected the OVD into the $\mathrm{MH}$ to gently lift the margins around the hole thus allowing the free large flap to be placed and fixed under the $\mathrm{MH}$ edges [91]. Other studies had incorporated PFC to harvest and stabilize autologous ILM intraoperatively with relative success in anatomical and visual outcomes [56, 92]. The authors also cautioned the risk of retinal injury as the flap may get stuck and difficult to dislodge from the forceps and the free ILM flap's orientation can be difficult to be identified intraoperatively [56].

Besides the difficulty in repositioning the free ILM flap, surgeons may find it challenging to harvest the right size for the graft to avoid redundancy or inadequate coverage of the $\mathrm{MH}$. Lowering the infusion pressure during transplantation, down to $10 \mathrm{mmHg}$, can assist in securing the free flap inside the $\mathrm{MH}$ to prevent excessive movements from the turbulence [93]. Other authors recommended performing the gas-fluid exchange at the surface of fluid level with passive aspirations toward the end to prevent the flap displacement. Fung et al. demonstrated that transposition and tucking autologous ILM without stabilization aids yielded an $87.5 \% \mathrm{MH}$ closure rate with a significant mean VA gain of 1.13 lines in cases with refractory MHs although it harbored risk of inflicting trauma to the RPE [94].

\section{Autologous lens capsular flap transplantation (LCFT)}

If autologous ILM free flap is not readily available due to prior wider ILM removal or difficulty in obtaining the fragile peripheral ILM, Chen et al. proposed 
transplantation of lens capsular flaps to close refractory MHs. Lens capsule has a higher density than ILM; thus, manipulating it during surgery is more convenient as it gravitationally settles down over the MHs once released from micro-forceps. There was no statistically significant difference in the visual acuity improvement in anterior and posterior capsule groups, but a better anatomical closure rate was seen in the former group as the anterior flap was more rigid than the posterior flap. The concomitant cataract surgery might affect the actual visual acuity outcome of the anterior group. Nonetheless, LCFT positively impacts the visual function as visual improvement was also seen in the posterior capsule group without the cataract surgery [95].

Authors of a long-term study with a mean follow-up of 18.5 months demonstrated that LCFT is ideal as a firstline treatment for refractory MHs. The study investigated LCFT either autologous or allogenic in origin in $48 \mathrm{MHs}$ with a mean diameter of $1102 \mu \mathrm{m}$. In this study, $96 \%$ of MHs were closed with significantly improved median visual acuity from baseline $1.79 \log$ MAR to postoperative $1.00 \log$ MAR. The authors also recommended adjunct whole blood to tamponade the LCFT to prevent its displacement [96].

\section{Autologous neurosensory retinal transplant (ART)}

Harvesting and transplanting the free ILM flap can be challenging in myopic MHs due to poor staining of the ILM and fragile nature of the eye from weakened retinalposterior pole adherence, which is secondary to RPE and choroidal atrophy and posterior staphyloma [86, 97]. Grewal et al. demonstrated the successful use of neurosensory retinal graft to close refractory myopic MHs with RD in a complex ocular case as autologous lens capsule and ILM free flaps were unattainable for transplantation. A 2 DD ART graft was harvested from the neurosensory retinal site superior to the superotemporal arcade, and the graft site was secured by endolaser and endo-diathermy. The graft was stabilized in situ by perfluoro- $n$-Octane heavy liquid (PFCL) followed by direct PFCL-silicone oil exchange. MH was observed closed at 1 -week with gradual improvement in visual acuity, visual distortion, and scotoma size. These improvements were also supported by an increase in retinal sensitivity from $7.5 \mathrm{~dB}$ to $12.3 \mathrm{~dB}$ at 3 months while the graft site showed no evidence of ERM and RD. The authors reiterated that i-ILM flap is not indicated in refractory MHs that had failed primary ILM peel and PPV [98].

A multicenter retrospective study showed that $88 \%$ of 41 refractory MHs with an MBD of $1468.1 \pm 656.4 \mu \mathrm{m}$ were closed with ART and 52.3\% of the closed MHs showed visual improvement [99]. Other case reports demonstrated successful $\mathrm{MH}$ closure following ART in persistent FTMH and complicated $\mathrm{MH}$ in recurrent myopic RD with visual acuity gain from BCVA preoperative $20 / 800$ and light perception to postoperative $20 / 100$ and $20 / 400$, respectively $[100,101]$. A recent study demonstrated ART closed $76.92 \%$ of refractory MHs with an MBD of $1615.38 \pm 689.19 \mu \mathrm{m}$ and at one year, six of the closed MHs achieved full restoration of the myoid/ellipsoid layer. However, there was no statistically significant visual gain observed, and one eye developed posterior vitreoretinopathy and ERM from the ART harvested site [102]. Although these changes did not affect the patient's visual acuity, a long-term study is warranted to investigate ART's safety profile as only transient graft edema was only reported in other studies [99, 100, 103].

ART showed anatomic integration, according to Grewal et al., as significantly reduced EZ and ELM defects were demonstrated on the OCT and integration of the graft within the adjoining retina and its migration to the $\mathrm{MH}$ [99]. A small series of four eyes had shown a better EZ and ELM defects recovery in cases whereby the grafts were placed under the edges of the $\mathrm{MH}$ compared to the epiretinally positioned grafts as its closer contact with the RPE promotes improved tissue integration and photoreceptor survival [104]. Tabandeh studied the graft's vascular profile after large ART in two giant MHs with an MBD of $2914 \mu \mathrm{m}$. Like other older studies that had demonstrated the graft's anatomical integration into the surrounding retina, Tabandeh also observed the graft's vascular reperfusion on angiography imaging at the earliest 6 weeks. He argued that the ischemic 5 DD retinal graft stimulated adequate angiogenesis that promoted anastomosis between the graft and the retina. Subsequently, the angiogenic drive diminished as reperfusion took place and prevented exaggerated angiogenesis response. He also described various stages of the graft's physiological changes in correlation to MH's size and visual improvement [105].

Displacement of the neurosensory retinal flap can still occur during surgery and postoperatively. Thus, Grewal et al. recommended harvesting and maneuvering the graft under the PFCL, which can be left as a safe shortterm tamponade for 1 to 2 weeks although it requires repeat surgery for its removal [99]. Other smaller studies had successfully used deuterium oxide, autologous blood clots, and viscoelastic to secure the retinal graft in situ in managing refractory $\mathrm{MHs}$, including recurrent MHRD [103, 106, 107]. While positioning ILM or lens capsule flaps inside MHs are at risk of iatrogenic trauma, Grewal et al. justified that the ART minimizes the surgical trauma as it can be positioned over the MH due to its thicker and sturdier graft [99]. 
Overall, the neurosensory retinal free flap created a barrier between the vitreous and the subretinal space thus to let the subretinal fluid excretion by the RPE and to scaffold glial repair, though the exact mechanism of the graft's structural integration is yet to be clarified [98, $103,106]$. However, ART may not be indicated if the retinal graft is not viable for harvesting including ischemic or inflamed retinae, neovascularization, or extensive scarring of the chorioretinae.

\section{Human amniotic membrane (hAM) transplantation}

hAM has been utilized in ocular surface diseases and provides a biological scaffolding for conjunctival growth. A porcine study supported the finding of hAM in promoting the growth of RPE tissue in the subretinal space [108]. Rizzo et al. first demonstrated filling of the MHs with layers of the neurosensory retina in all eight eyes at one week following transplanted hAM into the subretinal space via the $\mathrm{MH}$. All closed refractory $\mathrm{MHs}$ had improvement in the visual acuity from pre-operative 1.48 $\log$ MAR to $0.48 \log$ MAR at six months. The authors also successfully used hAM to treat six retinal breaks in complex RD cases in this prospective series [109].

While in highly myopic MHs with $\mathrm{AL}>30 \mathrm{~mm}$, dislocated hAM plug may occur after intraocular gas reabsorption and require repeat procedure as seen in one eye from a series of 16 eyes, MHs closure was readily seen on OCT examination in 15 eyes at two weeks. Although a slight improvement was observed in their visual acuities postoperatively, the $100 \%$ anatomical closure in all eyes remains a success given a potential complicating $R D$ in persistent MHs in myopic eyes [110]. Both studies demonstrated that hAM plugs successfully induce growth of retina into organized layers that leads to recovery of original retinal function virtually $[109,110]$.

A meta-analysis demonstrated that the hAM-treated group achieved at least double the visual gain compared to other reported techniques for refractory MHs. This is likely attributed to the placement of the hAM compared to other tissue grafts as it was transplanted under the margins of the FTMHs and in the subretinal space. The close contact between the RPE and the graft protected the $\mathrm{MH}$ from vitreous and also promoted RPE proliferation and retinal restoration as per in vitro studies [108, 111]. Nevertheless, the anatomical success rate between hAM and other autologous ILM and non-ILM grafts transplants was similar to close refractory MHs [112].

\section{Others}

Multiple studies had investigated subretinal infusion, $\mathrm{MH}$ hydrodissection, and relaxing retinotomies to close refractory MHs [112-116]. The goals are to increase retinal tissue compliance and to mobilize the edges of the MHs, thus improving the likelihood of the refractory $\mathrm{MHs}$ to close and attain improved visual outcomes. The outpatient fluid-gas exchange has been studied by additional intraocular gas top-up and better results were achieved with the long-acting gas compared to the shortacting gas [117]. It was more feasible, cost effective, and tolerable for patients. Heavy silicone oil like Densiron 68 had been used in the retreatment of persistent and recurrent MHs and it yielded better surgical outcomes compared to gas and silicone oil endotamponade [118]. It also has a better safety profile than silicone oil [30]. The experimental use of MSCs in treating large and recalcitrant MHs was studied by Zhang et al. with promising results although a larger sample size and long-term study are warranted [119].

\section{Conclusion}

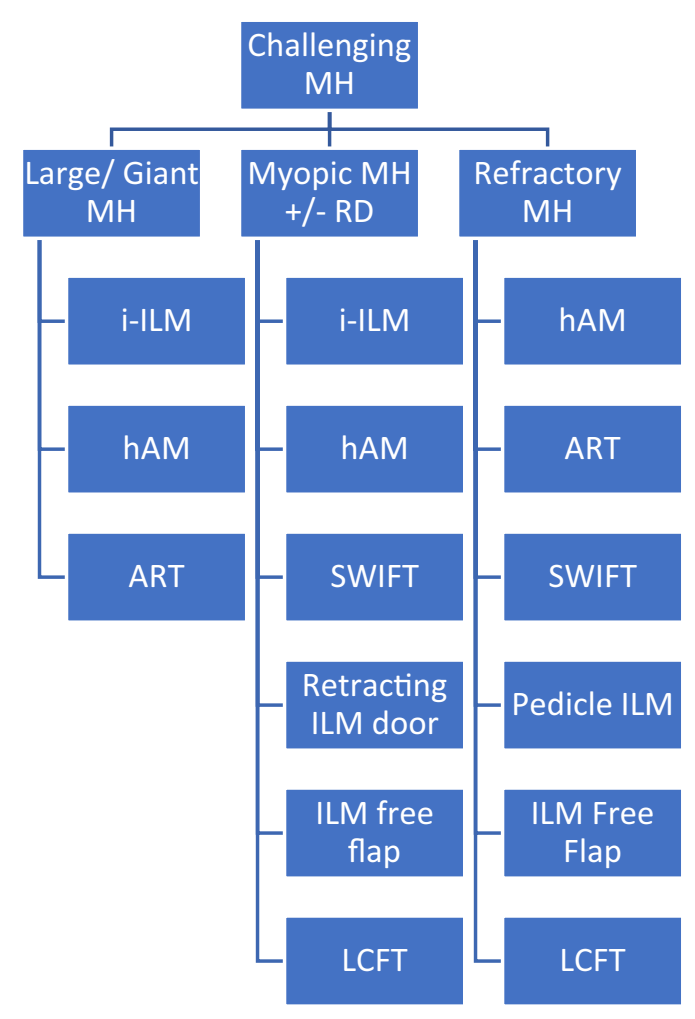

As all MHs do not happen equally, there is no definitive method or technique to propose the best method to close difficult MHs. There is extensive evidence supporting the i-ILM flap as the primary treatment for large idiopathic MHs and highly myopic MHs. With the growing data on the experimental use of various surgical techniques and discoveries of novel adjuvants that exert additional biological advantages on top of the scaffolding to close the $\mathrm{MHs}$, it is exciting to see the vast alternatives besides i-ILM flap to treat the devastating disease. There is a shift 
of trend from ILM peeling to ILM flap manipulation to preserving as much ILM for future utilization if required.

The patient's wellbeing must be factored-in in considering if it is worthwhile to operate on challenging $\mathrm{MH}$ with poor visual baseline or even reoperating in MHs with unfavorable outcomes. Patients will be subjected to a stricter ordeal of postoperative posturing and it costs them more time, expenditure, and effort. However, it can be argued that there is a very limited window to stabilize vision or to prevent further deterioration of macular thus there may be a benefit to surgery, even if the functional outcome is not expected to improve greatly. An opened refractory myopic $\mathrm{MH}$ imposes the risk of recurrent RD, which can lead to devastating sequelae if left untreated.

The end goals of $\mathrm{MH}$ surgery are to remove or mitigate the forces that "open" the $\mathrm{MH}$, to bridge or to provide scaffolds to join the edges of the MH together, thereby closing the $\mathrm{MH}$, in the hope of improving vision. Visual success is not proportionally commensurate with anatomical success as visual gain seen in extra-large and chronic MHs was deemed inferior even after successful closure, highlighting the importance of determining the pre-operative size of the $\mathrm{MH}$ as one of the predictive factors in the determination of visual benefit postoperatively. The expanding niche in improvising and inventing surgical techniques with more novel adjuvants will likely improve the surgical outcomes even in MHs with the worst prognosis.

Overall, most studies have similar limitations, including selection bias and small sample size, and they require longer follow-up times to elucidate any potential Type II errors. Furthermore, all the above techniques that were collectively discussed may not find unanimous support of all the retinal surgeons worldwide, as different surgeons may find specific techniques work better in their care and that patient's selection and postoperative expectations may also be the deciding factors in choosing the preferred approach in the management of these challenging MHs.

\begin{abstract}
Abbreviations
MHs: Macular holes; FTMHs: Full-thickness macular holes; ILM: Internal limiting membrane; RPE: Retinal pigment epithelium; PVD: Posterior vitreous detachment; ERM: Epiretinal membrane; RD: Retinal detachment; EZ: Ellipsoid zone; ELM: External limiting membrane; PPV: Pars plana vitrectomy; RCTs: Randomized controlled trials; ICG: Indocyanine green; TB: Trypan blue; BBG: Brilliant blue-G; DD: Disc diameter; DONFL: Dissociated optic nerve fiber layer; BCVA: Best-corrected visual acuity; Log MAR: Logarithm of the minimum angle of resolution; hAM: Human amniotic membrane; APC: Autologous platelet concentrate; OCT: Optical coherence tomography; TGF- $\beta_{2}$ : Transforming growth factor beta 2; SO: Silicone oil.
\end{abstract}

Acknowledgements

We are grateful for the manuscript proofreading by Alfreda.

\section{Authors' contributions}

MA conceptualized the work, reviewed and analyzed the relevant articles, and prepared the manuscript. LL reviewed the manuscript overall. MA and LL approved the final version of the manuscript.

\section{Funding}

Open Access funding provided by Universiti Malaysia Sarawak. The authors received no financial support for the research, authorship, and/or publication of this review article.

\section{Availability of data and materials \\ Not applicable.}

\section{Declarations}

Ethics approval and consent to participate

Not applicable.

Consent for publication

Not applicable.

\section{Competing interests}

The authors declared no potential conflicts of interest with respect to the research, authorship, and/or publication of this article.

Received: 29 April 2021 Accepted: 5 December 2021

Published online: 20 December 2021

\section{References}

1. Knapp H. Ueber Isolierte Zerreissungen der Aderhaut infolge von Traumen auf dem Augapfel. Arch Augenheilkd. 1869;1:6-29.

2. Zhang $L, L i X$, Yang $X$, Shen $Y, W u M$. Internal limiting membrane insertion technique combined with nerve growth factor injection for large macular hole. BMC Ophthalmol. 2019;19(1):247.

3. McCannel CA, Ensminger JL, Diehl NN, Hodge DN. Population-based incidence of macular holes. Ophthalmology. 2009;1 16(7):1366-9.

4. Ezra E. Idiopathic full thickness macular hole: natural history and pathogenesis. Br J Ophthalmol. 2001;85(1):102.

5. Spiteri Cornish K, Lois N, Scott N, Burr J, Cook J, Boachie C, et al. Vitrectomy with internal limiting membrane (ILM) peeling versus vitrectomy with no peeling for idiopathic full-thickness macular hole (FTMH). Cochrane Database Syst Rev. 2013;6:cd009306.

6. Cao JL, Kaiser PK. Surgical management of recurrent and persistent macular holes: a practical approach. Ophthalmol Ther. 2021;10(4):1137-53.

7. Duker JS, Kaiser PK, Binder S, de Smet MD, Gaudric A, Reichel E, et al. The International Vitreomacular Traction Study Group classification of vitreomacular adhesion, traction, and macular hole. Ophthalmology. 2013;120(12):2611-9.

8. Michalewska Z, Michalewski J, Adelman RA, Nawrocki J. Inverted internal limiting membrane flap technique for large macular holes. Ophthalmology. 2010;117(10):2018-25.

9. Yamashita T, Sakamoto T, Terasaki H, Iwasaki M, Ogushi Y, Okamoto $F$, et al. Best surgical technique and outcomes for large macular holes: retrospective multicentre study in Japan. Acta Ophthalmol. 2018;96(8):e904-10

10. Imai M, lijima H, Gotoh T, Tsukahara S. Optical coherence tomography of successfully repaired idiopathic macular holes. Am J Ophthalmol. 1999;128(5):621-7.

11. Ch'ng SW, Patton N, Ahmed M, Ivanova T, Baumann C, Charles S, et al. The manchester large macular hole study: is it time to reclassify large macular holes? Am J Ophthalmol. 2018;195:36-42.

12. Ip MS, Baker BJ, Duker JS, Reichel E, Baumal CR, Gangnon R, et al. Anatomical outcomes of surgery for idiopathic macular hole as determined by optical coherence tomography. Arch Ophthalmol. 2002;120(1):29-35. 
13. Jaycock PD, Bunce C, Xing W, Thomas D, Poon W, Gazzard G, et al. Outcomes of macular hole surgery: implications for surgical management and clinical governance. Eye (Lond). 2005;19(8):879-84.

14. Willis AW, Garcia-Cosio JF. Macular hole surgery. Comparison of longstanding versus recent macular holes. Ophthalmology. 1996;103(11):1811-4.

15. Wu TT, Kung YH. Comparison of anatomical and visual outcomes of macular hole surgery in patients with high myopia vs. non-high myopia: a case-control study using optical coherence tomography. Graefes Arch Clin Exp Ophthalmol. 2012;250(3):327-31.

16. Suda K, Hangai M, Yoshimura N. Axial length and outcomes of macular hole surgery assessed by spectral-domain optical coherence tomography. Am J Ophthalmol. 2011;151(1):118-27.e1.

17. Finn AP, Mahmoud TH. Internal limiting membrane retracting door for myopic macular holes. Retina. 2019;39(Suppl 1):S92-4.

18. Alkabes M, Pichi F, Nucci P, Massaro D, Dutra Medeiros M, Corcostegui $B$, et al. Anatomical and visual outcomes in high myopic macular hole (HM-MH) without retinal detachment: a review. Graefes Arch Clin Exp Ophthalmol. 2014;252(2):191-9.

19. Michalewska Z, Michalewski J, Dulczewska-Cichecka K, Adelman RA, Nawrocki J. Temporal inverted internal limiting membrane flap technique versus classic inverted internal limiting membrane flap technique: a comparative study. Retina. 2015;35(9):1844-50.

20. Andrew N, Chan WO, Tan M, Ebneter A, Gilhotra JS. Modification of the inverted internal limiting membrane flap technique for the treatment of chronic and large macular holes. Retina. 2016;36(4):834-7.

21. Ghassemi F, Khojasteh H, Khodabande A, Dalvin LA, Mazloumi M, RiaziEsfahani $\mathrm{H}$, et al. Comparison of three different techniques of inverted internal limiting membrane flap in treatment of large idiopathic fullthickness macular hole. Clin Ophthalmol. 2019;13:2599-606.

22. Kelly NE, Wendel RT. Vitreous surgery for idiopathic macular. holes Results of a pilot study. Arch Ophthalmol. 1991;109(5):654-9.

23. Smiddy WE, Feuer W, Cordahi G. Internal limiting membrane peeling in macular hole surgery. Ophthalmology. 2001;108(8):1471-6 (discussion 7-8).

24. Mahmoud TH, Thompson JT. The treatment of difficult macular holes. Ophthalmol Retina. 2021;5(4):315-6.

25. Kang SW, Ahn K, Ham DI. Types of macular hole closure and their clinical implications. Br J Ophthalmol. 2003;87(8):1015-9.

26. Hillenkamp J, Kraus J, Framme C, Jackson TL, Roider J, Gabel V-P, et al. Retreatment of full-thickness macular hole: predictive value of optical coherence tomography. Br J Ophthalmol. 2007;91(11):1445-9.

27. Ooto S, Akagi T, Kageyama R, Akita J, Mandai M, Honda Y, et al. Potential for neural regeneration after neurotoxic injury in the adult mammalian retina. Proc Natl Acad Sci U S A. 2004;101(37):13654-9.

28. Fernandez-Bueno I, Pastor JC, Gayoso MJ, Alcalde I, Garcia MT. Müller and macrophage-like cell interactions in an organotypic culture of porcine neuroretina. Mol Vis. 2008;14:2148-56.

29. Michalewska Z, Nawrocki J. Macular hole surgery in a patient who cannot maintain facedown positioning. Case Rep Ophthalmol. 2013;4(1):1-6.

30. Tam ALC, Yan P, Gan NY, Lam WC. The current surgical management of large, recurrent, or persistent macular holes. Retina. 2018;38(7):1263-75.

31. Ittarat M, Somkijrungroj T, Chansangpetch S, Pongsachareonnont P. Literature review of surgical treatment in idiopathic full-thickness macular hole. Clin Ophthalmol. 2020;14:2171-83.

32. Cheng L, Azen SP, El-Bradey MH, Toyoguchi M, Chaidhawangul S, Rivero $M E$, et al. Effects of preoperative and postoperative epiretinal membranes on macular hole closure and visual restoration. Ophthalmology. 2002;109(8):1514-20.

33. Rahimy E, McCannel CA. Impact of internal limiting membrane peeling on macular hole reopening: a systematic review and meta-analysis. Retina. 2016;36(4):679-87.

34. Vaziri K, Schwartz SG, Kishor KS, Fortun JA, Moshfeghi AA, Smiddy WE, et al. Rates of reoperation and retinal detachment after macular hole surgery. Ophthalmology. 2016;123(1):26-31.

35. Kim SS, Smiddy WE, Feuer WJ, Shi W. Outcomes of sulfur hexafluoride (SF6) versus perfluoropropane (C3F8) gas tamponade for macular hole surgery. Retina. 2008;28(10):1408-15.
36. Modi A, Giridhar A, Gopalakrishnan M. Sulfurhexafluoride (SF6) versus perfluoropropane (C3F8) gas as tamponade in macular hole surgery. Retina. 2017;37(2):283-90.

37. Briand S, Chalifoux E, Tourville E, Bourgault S, Caissie M, Tardif Y, et al. Prospective randomized trial: outcomes of $\mathrm{SF}_{6}$ versus $\mathrm{C}_{3} \mathrm{~F}_{8}$ in macular hole surgery. Can J Ophthalmol. 2015;50(2):95-100.

38. Pasu S, Bell L, Zenasni Z, Lanz D, Simmonds IA, Thompson A, et al. Facedown positioning following surgery for large full-thickness macular hole: a multicenter randomized clinical trial. JAMA Ophthalmol. 2020;138(7):725-30.

39. Eckardt C, Eckardt U, Groos S, Luciano L, Reale E. Removal of the internal limiting membrane in macular holes. Clinical and morphological findings. Ophthalmologe. 1997;94(8):545-51.

40. Christensen UC, Krøyer K, Sander B, Larsen M, Henning V, Villumsen $J$, et al. Value of internal limiting membrane peeling in surgery for idiopathic macular hole stage 2 and 3: a randomised clinical trial. $\mathrm{Br} J$ Ophthalmol. 2009;93(8):1005-15.

41. Kwok AK, Lai TY, Wong VW. Idiopathic macular hole surgery in Chinese patients: a randomised study to compare indocyanine green-assisted internal limiting membrane peeling with no internal limiting membrane peeling. Hong Kong Med J. 2005;11(4):259-66.

42. Lois N, Burr J, Norrie J, Vale L, Cook J, McDonald A, et al. Internal limiting membrane peeling versus no peeling for idiopathic full-thickness macular hole: a pragmatic randomized controlled trial. Invest Ophthalmol Vis Sci. 2011;52(3):1586-92.

43. Tognetto D, Grandin R, Sanguinetti G, Minutola D, Di Nicola M, Di Mascio $R$, et al. Internal limiting membrane removal during macular hole surgery: results of a multicenter retrospective study. Ophthalmology. 2006;113(8):1401-10.

44. Mester $\vee$, Kuhn F. Internal limiting membrane removal in the management of full-thickness macular holes. Am J Ophthalmol. 2000;129(6):769-77.

45. Kumagai K, Furukawa M, Ogino N, Uemura A, Demizu S, Larson E. Vitreous surgery with and without internal limiting membrane peeling for macular hole repair. Retina. 2004;24(5):721-7.

46. Haritoglou C, Reiniger IW, Schaumberger M, Gass CA, Priglinger SG, Kampik A. Five-year follow-up of macular hole surgery with peeling of the internal limiting membrane: update of a prospective study. Retina. 2006;26(6):618-22.

47. Al Sabti K, Kumar N, Azad RV. Extended internal limiting membrane peeling in the management of unusually large macular holes. Ophthalmic Surg Lasers Imaging. 2009;40(2):185-7.

48. Valldeperas $X$, Wong D. Is it worth reoperating on macular holes? Ophthalmology. 2008;115(1):158-63.

49. Moisseiev E, Fabian ID, Moisseiev J, Barak A. Outcomes of repeated pars plana vitrectomy for persistent macular holes. Retina. 2013;33(6):1137-43

50. D'Souza MJ, Chaudhary V, Devenyi R, Kertes PJ, Lam WC. Re-operation of idiopathic full-thickness macular holes after initial surgery with internal limiting membrane peel. Br J Ophthalmol. 2011;95(11):1564-7.

51. Che X, He F, Lu L, Zhu D, Xu X, Song X, et al. Evaluation of secondary surgery to enlarge the peeling of the internal limiting membrane following the failed surgery of idiopathic macular holes. Exp Ther Med. 2014;7(3):742-6.

52. Haritoglou C, Gass CA, Schaumberger M, Ehrt O, Gandorfer A, Kampik A. Macular changes after peeling of the internal limiting membrane in macular hole surgery. Am J Ophthalmol. 2001;132(3):363-8.

53. Clark A, Balducci N, Pichi F, Veronese C, Morara M, Torrazza C, et al. Swelling of the arcuate nerve fiber layer after internal limiting membrane peeling. Retina. 2012;32(8):1608-13.

54. Tadayoni R, Paques M, Massin P, Mouki-Benani S, Mikol J, Gaudric A. Dissociated optic nerve fiber layer appearance of the fundus after idiopathic epiretinal membrane removal. Ophthalmology. 2001;108(12):2279-83.

55. Mitamura Y, Ohtsuka K. Relationship of dissociated optic nerve fiber layer appearance to internal limiting membrane peeling. Ophthalmology. 2005;112(10):1766-70.

56. Park SW, Pak KY, Park KH, Kim KH, Byon IS, Lee JE. Perfluoro-n-octane assisted free internal limiting membrane flap technique for recurrent macular hole. Retina. 2015;35(12):2652-6. 
57. Shin MK, Park KH, Park SW, Byon IS, Lee JE. Perfluoro-n-octane-assisted single-layered inverted internal limiting membrane flap technique for macular hole surgery. Retina. 2014;34(9):1905-10.

58. Chou HD, Chong YJ, Teh WM, Chen KJ, Liu L, Chen YP, et al. Nasal or temporal internal limiting membrane flap assisted by sub-perfluorocarbon viscoelastic injection for macular hole repair. Am J Ophthalmol. 2021;223:296-305.

59. Hu Z, Qian H, Fransisca S, Gu X, Ji J, Wang J, et al. Minimal internal limiting membrane peeling with ILM flap technique for idiopathic macular holes: a preliminary study. BMC Ophthalmol. 2020;20(1):228.

60. Karalezli A, Kaderli ST, Sul S. Macular microvasculature differences in patients with macular hole after vitrectomy with internal limiting membrane removal or single-layered temporal inverted flap technique. Eye. 2021;35(10):2746-53.

61. Kaluzny JJ, Zabel P, Kaluzna M, Lamkowski A, Jaworski D, Woznicki K, et al. Macular sensitivity in the area of internal limiting membrane peeling in eyes after pars plana vitrectomy with the temporal inverted internal limiting membrane flap technique for a full-thickness macular hole. Retina. 2021;41(8):1627-34.

62. Faria MY, Proença H, Ferreira NG, Sousa DC, Neto E, Marques-Neves C. Inverted internal limiting membrane flap techniques and outer retinal layer structures. Retina. 2020;40(7):1299-305.

63. Cacciamani A, Gelso A, Di Nicola M, Scarinci F, Ripandelli G, Costagliola C, et al. Inverted ILM-flap techniques variants for macular hole surgery: randomized clinical trial to compare retinal sensitivity and fixation stability. Sci Rep. 2020;10(1):15832.

64. Rossi T, Gelso A, Costagliola C, Trillo C, Costa A, Gesualdo C, Ripandelli G (2017) Macular hole closure patterns associated with different internal limiting membrane flap techniques. Graefes Arch Clin Exp Ophthalmol 255(6) 1073-1078. https://doi.org/10.1007/s00417-017-3598-9

65. Park JH, Lee SM, Park SW, Lee JE, Byon IS. Comparative analysis of large macular hole surgery using an internal limiting membrane insertion versus inverted flap technique. Br J Ophthalmol. 2019;103(2):245.

66. Rizzo S, Tartaro R, Barca F, Caporossi T, Bacherini D, Giansanti F. Internal limiting membrane peeling versus inverted flap technique for treatment of full-thickness macular holes: a comparative study in a large series of patients. Retina. 2018;38(Suppl 1):S73-8.

67. Gu C, Qiu Q. Inverted internal limiting membrane flap technique for large macular holes: a systematic review and single-arm meta-analysis. Graefes Arch Clin Exp Ophthalmol. 2018;256(6):1041-9.

68. Manasa S, Kakkar P, Kumar A, Chandra P, Kumar V, Ravani R. Comparative evaluation of standard ILM peel with inverted ILM flap technique in large macular holes: a prospective, randomized study. Ophthalmic Surg Lasers Imaging Retina. 2018;49(4):236-40.

69. Kannan NB, Kohli P, Parida H, Adenuga OO, Ramasamy K. Comparative study of inverted internal limiting membrane (ILM) flap and ILM peeling technique in large macular holes: a randomized-control trial. BMC Ophthalmol. 2018;18(1):177.

70. Narayanan R, Singh SR, Taylor S, Berrocal MH, Chhablani J, Tyagi M, et al. Surgical outcomes after inverted internal limiting membrane flap versus conventional peeling for very large macular holes. Retina. 2019;39(8):1465-9.

71. Yuan J, Zhang L-L, Lu Y-J, Han M-Y, Yu A-H, Cai X-J. Vitrectomy with internal limiting membrane peeling versus inverted internal limiting membrane flap technique for macular hole-induced retinal detachment: a systematic review of literature and meta-analysis. BMC Ophthalmol. 2017;17(1):219.

72. Hu X-T, Pan Q-T, Zheng J-W, Zhang Z-D. Foveal microstructure and visual outcomes of myopic macular hole surgery with or without the inverted internal limiting membrane flap technique. Br J Ophthalmol. 2019;103(10):1495.

73. Michalewska Z, Michalewski J, Dulczewska-Cichecka K, Nawrocki J. Inverted internal limiting membrane flap technique for surgical repair of myopic macular holes. Retina. 2014;34(4):664-9.

74. Marques RE, Sousa DC, Leal I, Faria MY, Marques-Neves C. Complete ILM peeling versus inverted flap technique for macular hole surgery: a meta-analysis. Ophthalmic Surg Lasers Imaging Retina. 2020;51(3):187-a2

75. Shen Y, Lin X, Zhang L, Wu M. Comparative efficacy evaluation of inverted internal limiting membrane flap technique and internal limiting membrane peeling in large macular holes: a systematic review and meta-analysis. BMC Ophthalmol. 2020;20(1):14.

76. Ramtohul P, Parrat E, Denis D, Lorenzi U. Inverted internal limiting membrane flap technique versus complete internal limiting membrane peeling in large macular hole surgery: a comparative study. BMC Ophthalmol. 2020;20(1):11.

77. Baumann C, Kaye S, lannetta D, Sultan Z, Dwivedi R, Pearce I. Effect of inverted internal limiting membrane flap on closure rate, postoperative visual acuity, and restoration of outer retinal layers in primary idiopathic macular hole surgerY. Retina. 2020;40(10):1955-63.

78. Iwasaki M, Kinoshita T, Miyamoto $\mathrm{H}$, Imaizumi H. Influence of inverted internal limiting membrane flap technique on the outer retinal layer structures after a large macular hole surgery. Retina. 2019;39(8):1470-7.

79. Hirata A, Mine K, Hayashi K. Contractility of temporal inverted internal limiting membrane flap after vitrectomy for macular hole. Sci Rep. 2021;11(1):20035

80. Tabandeh H, Morozov A, Rezaei KA, Boyer DS. Superior wide-base internal limiting membrane flap transposition for macular holes: flap status and outcomes. Ophthalmology Retina. 2021;5(4):317-23.

81. Tabandeh $\mathrm{H}$. Fluorescence imaging of the ILM flap following $\mathrm{MH}$ surgery. Am J Ophthalmol Case Rep. 2021;24:101203.

82. Hu Z, Ye X, Lv X, Liang K, Zhang W, Chen X, et al. Non-inverted pedicle internal limiting membrane transposition for large macular holes. Eye. 2018:32(9):1512-8.

83. da Silva Tavares Neto JE, Coelho IN, Jorge R, Isaac DLC, de Ávila MP. Pedicle internal limiting membrane flap technique for very large macular holes: a preliminary report. Int J Retina Vitreous. 2020;6:43.

84. Gekka T, Watanabe A, Ohkuma Y, Arai K, Watanabe T, Tsuzuki A, et al. Pedicle internal limiting membrane transposition flap technique for refractory macular hole. Ophthalmic Surg Lasers Imaging Retina. 2015;46(10):1045-6.

85. Marlow ED, Mahmoud TH. Current management strategies for atypical macular holes. Taiwan J Ophthalmol. 2021;11(3):221-31.

86. Morizane Y, Shiraga F, Kimura S, Hosokawa M, Shiode Y, Kawata T, et al. Autologous transplantation of the internal limiting membrane for refractory macular holes. Am J Ophthalmol. 2014;157(4):861-9.e1.

87. Rossi T, Trillo C, Ripandelli G. Autologous internal limiting membrane transplant for recurrent idiopathic macular holes. Eur J Ophthalmol. 2021;31(2):656-63.

88. Chen SN, Yang CM. Double internal limiting membrane insertion for macular hole-associated retinal detachment. J Ophthalmol. 2017;2017:3236516.

89. Chen SN, Hsieh YT, Yang CM. Multiple free internal limiting membrane flap insertion in the treatment of macular hole-associated retinal detachment in high myopia. Ophthalmologica. 2018;240(3):143-9.

90. Lee SM, Kwon HJ, Park SW, Lee JE, Byon IS. Microstructural changes in the fovea following autologous internal limiting membrane transplantation surgery for large macular holes. Acta Ophthalmol. 2018;96(3):e406-8.

91. Dai Y, Dong F, Zhang $X$, Yang Z. Internal limiting membrane transplantation for unclosed and large macular holes. Graefes Arch Clin Exp Ophthalmol. 2016;254(11):2095-9.

92. Ozdek S, Baskaran P, Karabas L, Neves PP. A modified perfluoro-noctane-assisted autologous internal limiting membrane transplant for failed macular hole reintervention: a case series. Ophthalmic Surg Lasers Imaging Retina. 2017; 48(5):416-20.

93. Giansanti F, Tartaro R, Caporossi T, Bacherini D, Savastano A, Barca F, et al. An internal limiting membrane plug and gas endotamponade for recurrent or persistent macular hole. J Ophthalmol. 2019;2019:6051724.

94. Fung NSK, Mak AKH, Yiu R, Wong IYH, Lam WC. Treatment of large, chronic and persistent macular hole with internal limiting membrane transposition and tuck technique. Int J Retina Vitreous. 2020;6(1):3.

95. Chen SN, Yang CM. Lens capsular flap transplantation in the management of refractory macular hole from multiple etiologies. Retina. 2016;36(1):163-70.

96. Peng J, Chen C, Zhang H, Zhang L, Liu J, Ren J, et al. Long-term surgical outcomes of lens capsular flap transplantation in the management of refractory macular hole. Retina. 2021;41(4):726-34.

97. Lai CC, Chen YP, Wang NK, Chuang LH, Liu L, Chen KJ, et al. Vitrectomy with internal limiting membrane repositioning and autologous blood 
for macular hole retinal detachment in highly myopic eyes. Ophthalmology. 2015;122(9):1889-98.

98. Grewal DS, Mahmoud TH. Autologous neurosensory retinal free flap for closure of refractory myopic macular holes. JAMA Ophthalmol. 2016;134(2):229-30

99. Grewal DS, Charles S, Parolini B, Kadonosono K, Mahmoud TH. Autologous retinal transplant for refractory macular holes: multicenter International Collaborative Study Group. Ophthalmology. 2019:126(10):1399-408.

100. De Giacinto C, D'Aloisio R, Cirigliano G, Pastore MR, Tognetto D. Autologous neurosensory retinal free patch transplantation for persistent full-thickness macular hole. Int Ophthalmol. 2019;39(5):1147-50.

101. Sousa Neves F, Braga J, Sepúlveda P, Bilhoto M. Refractory myopic retinal detachment and macular hole closure with autologous neurosensory retinal free flap. Case Rep Ophthalmol. 2019;10(2):160-4.

102. Rojas-Juárez S, Cisneros-Cortés J, Ramirez-Estudillo A, Velez-Montoya R. Autologous full-thickness retinal transplant for refractory large macular holes. Int J Retina Vitreous. 2020;6(1):60.

103. Chang YC, Liu PK, Kao TE, Chen KJ, Chen YH, Chiu WJ, et al. Management of refractory large macular hole with autologous neurosensory retinal free flap transplantation. Retina. 2020;40(11):2134-9.

104. Lumi X, Petrovic Pajic S, Sustar M, Fakin A, Hawlina M. Autologous neurosensory free-flap retinal transplantation for refractory chronic macular hole-outcomes evaluated by OCT, microperimetry, and multifocal electroretinography. Graefes Arch Clin Exp Ophthalmol. 2021;259(6):1443-53.

105. Tabandeh H. Vascularization and reperfusion of autologous retinal transplant for giant macular holes. JAMA Ophthalmol. 2020;138(3):305-9.

106. Ding C, Li S, Zeng J. Autologous neurosensory retinal transplantation for unclosed and large macular holes. Ophthalmic Res. 2019;61(2):88-93.

107. Wu AL, Chuang LH, Wang NK, Chen KJ, Liu L, Yeung L, et al. Refractory macular hole repaired by autologous retinal graft and blood clot. BMC Ophthalmol. 2018;18(1):213.

108. Kiilgaard JF, Scherfig E, Prause JU, la Cour M. Transplantation of amniotic membrane to the subretinal space in pigs. Stem Cells Int. 2012;2012:716968.

109. Rizzo S, Caporossi T, Tartaro R, Finocchio L, Franco F, Barca F, et al. A human amniotic membrane plug to promote retinal breaks repair and recurrent macular hole closure. Retina. 2019;39(Suppl 1):S95-s103.

110. Caporossi T, Pacini B, De Angelis L, Barca F, Peiretti E, Rizzo S. Human amniotic membrane to close recurrent, high myopic macular holes in pathologic myopia with axial length of $\geq 30 \mathrm{~mm}$. Retina. 2020;40(10):1946-54.

111. Capeáns C, Piñeiro A, Pardo M, Sueiro-López C, Blanco MJ, Domínguez $F$, et al. Amniotic membrane as support for human retinal pigment epithelium (RPE) cell growth. Acta Ophthalmol Scand. 2003;81(3):271-7.

112. Frisina R, Gius I, Tozzi L, Midena E. Refractory full thickness macular hole: current surgical management. Eye (Lond). 2021. https://doi.org/10. 1038/s41433-020-01330-y.

113. Gurelik G, Sul S, Klıç G, Özsaygııı C. A modified foveal advancement technique in the treatment of persistent large macular holes. Ophthalmic Surg Lasers Imaging Retina. 2017:48(10):793-8.

114. Szigiato AA, Gilani F, Walsh MK, Mandelcorn ED, Muni RH. Induction of macular detachment for the treatment of persistent or recurrent idiopathic macular holes. Retina. 2016;36(9):1694-8.

115. Felfeli T, Mandelcorn ED. Macular hole hydrodissection: surgical technique for the treatment of persistent, chronic, and large macular holes. Retina. 2019;39(4):743-52.

116. Shah AA, Thomas BJ, Yonekawa Y, Capone A Jr. Radial retinal incisions for complex pediatric traumatic macular holes. Retina. 2016;36(1):211-5.

117. Rao X, Wang NK, Chen YP, Hwang YS, Chuang LH, Liu IC, et al. Outcomes of outpatient fluid-gas exchange for open macular hole after vitrectomy. Am J Ophthalmol. 2013;156(2):326-33.e1.

118. Cillino S, Cillino G, Ferraro LL, Casuccio A. Treatment of persistently open macular holes with heavy silicone oil (Densiron 68) versus C2F6. A prospective randomized study. Retina. 2016;36(4):688-94.

119. Zhang X, Liu J, Yu B, Ma F, Ren X, Li X. Effects of mesenchymal stem cells and their exosomes on the healing of large and refractory macular holes. Graefes Arch Clin Exp Ophthalmol. 2018;256(11):2041-52.

\section{Publisher's Note}

Springer Nature remains neutral with regard to jurisdictional claims in published maps and institutional affiliations.
Ready to submit your research? Choose BMC and benefit from:

- fast, convenient online submission

- thorough peer review by experienced researchers in your field

- rapid publication on acceptance

- support for research data, including large and complex data types

- gold Open Access which fosters wider collaboration and increased citations

- maximum visibility for your research: over $100 \mathrm{M}$ website views per year

At BMC, research is always in progress.

Learn more biomedcentral.com/submissions 